\title{
Fractional Order Calculus: Basic Concepts and Engineering Applications
}

\author{
Ricardo Enrique Gutiérrez, ${ }^{1}$ João Maurício Rosário, ${ }^{1}$ \\ and José Tenreiro Machado ${ }^{2}$ \\ ${ }^{1}$ Department of Mechanical Engineering, UNICAMP, 13083-970 Campinas, Brazil \\ ${ }^{2}$ Department of Electrical Engineering, ISEP, 4200-072 Oporto, Portugal \\ Correspondence should be addressed to Ricardo Enrique Gutiérrez, rgutic@fem.unicamp.br
}

Received 7 October 2009; Revised 19 February 2010; Accepted 2 March 2010

Academic Editor: Katica R. (Stevanovic) Hedrih

Copyright (c) 2010 Ricardo Enrique Gutiérrez et al. This is an open access article distributed under the Creative Commons Attribution License, which permits unrestricted use, distribution, and reproduction in any medium, provided the original work is properly cited.

The fractional order calculus (FOC) is as old as the integer one although up to recently its application was exclusively in mathematics. Many real systems are better described with FOC differential equations as it is a well-suited tool to analyze problems of fractal dimension, with longterm "memory" and chaotic behavior. Those characteristics have attracted the engineers' interest in the latter years, and now it is a tool used in almost every area of science. This paper introduces the fundamentals of the FOC and some applications in systems' identification, control, mechatronics, and robotics, where it is a promissory research field.

\section{Introduction}

The fractional order calculus (FOC) was unexplored in engineering, because of its inherent complexity, the apparent self-sufficiency of the integer order calculus (IOC), and the fact that it does not have a fully acceptable geometrical or physical interpretation [1, 2]. Notwithstanding it represents more accurately some natural behavior related to different areas of engineering, and now it is used as a promissory tool in bioengineering $[3,4]$, viscoelasticity $[5,6]$, electronics $[7,8]$, robotics $[9-11]$, control theory $[12,13]$, and signal processing $[14,15]$ between others.

In the latter years FOC attracted engineers' attention, because it can describe the behavior of real dynamical systems in compact expressions, taking into account nonlocal characteristics like "infinite memory" [16-18]. Some instance are thermal diffusion phenomenon [19], botanical electrical impedances [20], model of love between humans [21], the relaxation of water on a porous dyke whose damping ratio is independent of the mass of 
moving water [22], and so forth. On the other hand, direction the behavior of a process with fractional order controllers would be an advantage, because the responses are not restricted to a sum of exponential functions, therefore a wide range of responses neglected by integer order calculus would be approached [23].

Bearing these ideas in mind, this paper is organized as follows. Section 2 presents the fundamentals and analytical definitions. Section 3 introduces several approaches to the solution of the 300-years-old problem of the geometrical interpretation of the FOC. Then in Section 4 some applications in systems' identification, control, and robotics are presented. Finally Section 5 introduces the main conclusions and future applications of FOC.

\section{Fractional Order Calculus (FOC)}

The intuitive idea of FOC is as old as IOC, it can be observed from a letter written by Leibniz to L'Hopital in 1695 [24]. It is a generalization of the IOC to a real or complex order [25]. Formally the real order generalization is introduced as follows:

$$
D^{\alpha}= \begin{cases}\frac{d^{\alpha}}{d t^{\alpha}} & \alpha>0 \\ 1 & \alpha=0 \\ \int_{a}^{t}(d \tau)^{-\alpha} & \alpha<0\end{cases}
$$

with $\alpha \in \Re$.

Its applications in engineering were delayed because FOC has multiple definitions $[18,26]$, there is not a simple geometrical interpretation and the IOC seems, at first sight, to be enough to solve engineering problems. However, many natural phenomena may be better described by a FOC formulation, because it takes into account the past behavior and it is compact when expressing high-order dynamics $[27,28]$. Some common definitions of FOC are listed as follows $[18,26]$ :

(i) Riemann-Liouville:

Integral:

$$
J_{c}^{\alpha} f(t)=\frac{1}{\Gamma(\alpha)} \int_{c}^{t} \frac{f(\tau)}{(t-\tau)^{1-\alpha}} d \tau
$$

Derivative:

$$
D^{\alpha} f(t)=\frac{d^{m}}{d t^{m}}\left[\frac{1}{\Gamma(m-\alpha)} \int_{0}^{t} \frac{f(\tau)}{(t-\tau)^{\alpha+1-m}} d \tau\right], \quad m \in \mathbb{Z}^{+}, m-1<\alpha \leq m,
$$


(ii) Grünwald-Letnikov:

Integral:

$$
D^{-\alpha}=\lim _{h \rightarrow 0} h^{\alpha} \sum_{m=0}^{(t-a) / h} \frac{\Gamma(\alpha+m)}{m ! \Gamma(\alpha)} f(t-m h),
$$

Derivative:

$$
D^{\alpha}=\lim _{h \rightarrow 0} \frac{1}{h^{\alpha}} \sum_{m=0}^{(t-a) / h}(-1)^{m} \frac{\Gamma(\alpha+1)}{m ! \Gamma(\alpha-m+1)} f(t-m h),
$$

(iii) Caputo:

$$
D_{*}^{\alpha} f(t)=\frac{1}{\Gamma(m-\alpha)} \int_{0}^{t} \frac{f^{(m)}(\tau)}{(t-\tau)^{\alpha+1-m}} d \tau
$$

(iv) Cauchy:

$$
f_{+}^{(\alpha)}=\int f(\tau) \frac{(t-\tau)^{-\alpha-1}}{\Gamma(-\alpha)} d \tau
$$

where the function $\Gamma(\alpha)$ is the generalization of factorial function [29] and it is defined as:

$$
\Gamma(x) \equiv \int_{0}^{\infty} y^{x-1} e^{-y} d y, \quad x>0
$$

or without a restriction for $x$

$$
\Gamma(x) \equiv \lim _{N \rightarrow \infty}\left[\frac{N ! N^{x}}{x(x+1)(x+2) \cdots(x+N)}\right] .
$$

We can choose one definition or another, depending on the application and the preference of the designer. In [26] the authors compare these definitions in applications of control and signals processing, finding that the Cauchy definition preserves some important frequency properties, that also exist in IOC simplifying the data's interpretation.

Some other tools of interest for engineers are the classical transforms of Laplace and Fourier, that are valid and used in order to simplify operations like convolution and can be applied to solve FOC differential equations. In FOC the Laplace transform is defined as [30]

$$
\mathcal{L}\left\{{ }_{0} D_{t}^{\alpha}\right\}=s^{\alpha} F(s)-\sum_{j=0}^{n-1} s^{j}\left[{ }_{0} D^{\alpha-j-1} f(0)\right], \quad n-1<\alpha<n, n \in \mathbb{Z} .
$$


As shown, this transform takes into account all initial conditions from the first to the $n$th -1 derivative. In practice, the Fourier transform can be achieved by replacing $s$ by $j w$ in (2.10).

In addition to the problem for which definition must be chosen based on its properties or implementation complexity, the engineers may know the implications of using a mathematical tool. An easy way to understand it, is by plotting it in a figure and seeing what is happening when it is applied. Pitifully for FOC it is a lack, but some approaches were proposed in the last decade, as will be presented in Section 3.

\section{Geometrical Interpretation}

In the case of integral order calculus, there is a well-accepted geometrical explanation which clearly relates some physical quantities, for example, instant rate of change of a function completely explains the relationship between concepts like position and speed of an object. Unfortunately, until the last decade there was no geometrical interpretation of the fractional order derivatives. One of them was proposed in [1] explaining FOC from a probabilistic point of view, using the Grünwald-Letnikov definition ((2.4) and (2.5)). If $\alpha$ is a value between 0 and 1 , and $\gamma$ is defined as

$$
r(\alpha, m)=(-1)^{m} \frac{\Gamma(\alpha+1)}{m ! \Gamma(\alpha-m+1)} .
$$

Then for $m=0$ we obtain $\gamma=1$, that is, the value of the function at evaluation time (present) appears with probability of 1 .

If $m>0$,

$$
-\sum_{m=1} \gamma(\alpha, m)=1
$$

For values of $m \neq 0$ the $\gamma$ value vanishes when the analysis point is far from the evaluation one. Therefore, the author suggests that the expression $-\sum_{m=1}^{\infty} \gamma(\alpha, m) x(t-m h)$ is the expected value of a random variable $X$, where

$$
P(X=x(m h))=|\gamma(\alpha, m)|, \quad m=1,2, \ldots, 0<\alpha<1 .
$$

Therefore the values near to the evaluation time (present) have more influence over the result than those that are far from it. This interpretation is shown in Figure 1.

A geometric interpretation based on Riemann-Louville-definition ((2.2) and (2.3)) was presented in [31]. This definition can be written as

$$
J_{0}^{\alpha}=\int_{0}^{t} f(\tau) d g(\tau)
$$

with

$$
g(\tau)=\frac{1}{\Gamma(\alpha+1)}\left[t^{\alpha}(t-\tau)^{\alpha}\right] .
$$




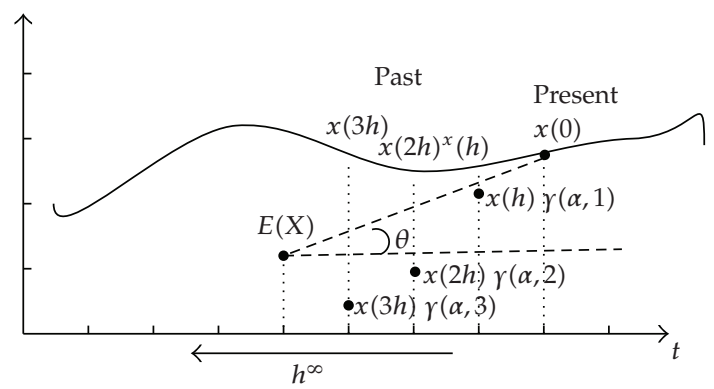

Figure 1: Tenreiro fractional order derivative interpretation. Here values near the evaluation point have a more significant effect over "the present" than others.

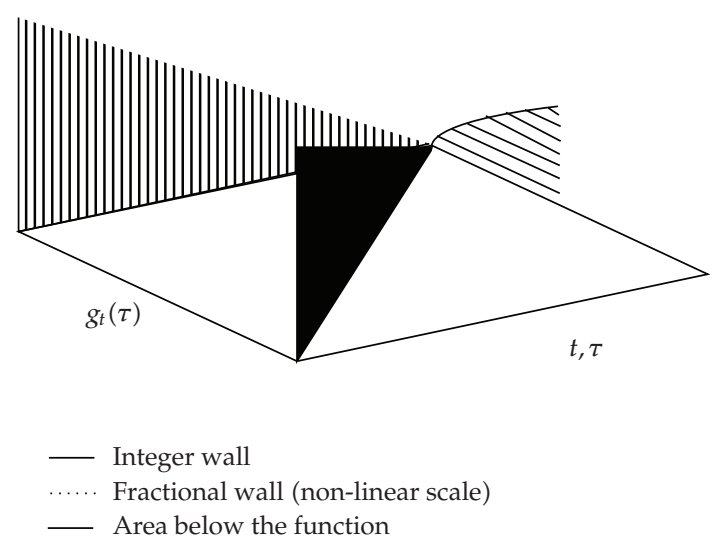

Figure 2: Podlubny fractional order derivative interpretation. $f(t)=t$ and $\alpha=0.3$ evaluated in the interval $[0,3]$. The fractional derivative is the projection of the area below the functions over a nonlinear time scale $g(\tau)$, with a deformation parameter $\alpha$ (order of the derivative).

With this information a tridimensional graph is drawn with axes $g(\tau), f(\tau)$, and $\tau$ as shown in Figure 2. The projection of the area below $f(t)$, over the plane $\langle\tau, f(\tau)\rangle$, is $\int_{0}^{t} f(\tau) d \tau$, the same as the integer integral definition. The projection of the area below the curve, over the plane $\langle f(\tau), g(\tau)\rangle$, is $\int_{0}^{t} f(\tau) d g(\tau)$. Note that it is the same definition as (3.4), that is, the integral of the function with a non homogeneous time scale that depends on the parameter $\alpha$.

Another geometrical interpretation, this time based fractal dimension was proposed in [32]. Here the author argues that the Riemman-Lioville (2.2) is the convolution of the function $f(t)$ with kernel:

$$
h_{\infty}=\frac{t^{\alpha-1}}{\Gamma(\alpha)}
$$

For $\alpha=0$, the function $h_{\infty}$ is undetermined. By increasing $\alpha$ (fractional integral case), the kernel $h_{\infty}$ takes into account the effect of the past values, weighting them (Figure 3 ). If $\alpha=1$ (integer integral case), then $J_{c}^{\alpha}$ has perfect memory and all the past is equally weighted. 


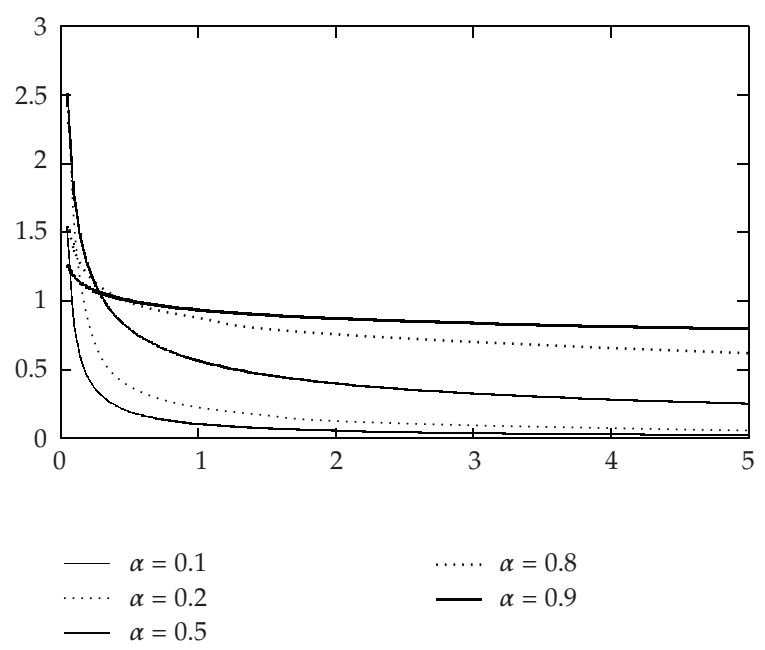

Figure 3: Function kernel $h_{\infty}$ evaluated for different $\alpha$ values. When convoluted with a function, $f(t)$ drives the weigh of the "memory" in the output.

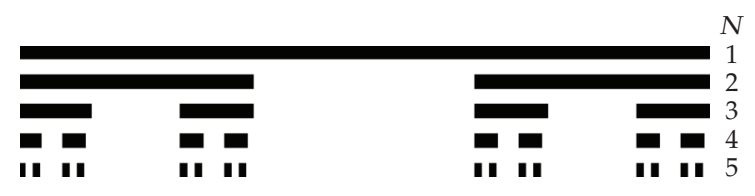

Figure 4: Cantor set with $\alpha=1 / 3$. Note that the density of the remaining segments is similar to the decay of the kernel function $h_{\infty}$.

In the derivative case $(-1<\alpha<0)$, the interpretation cannot be explicit obtained from (2.2). Using the Leibniz rule on (2.3) we obtain

$$
D^{\alpha} f(t)=\frac{f(0) t^{-\alpha}}{\Gamma(1-\alpha)}+\frac{1}{\Gamma(1-\alpha)} \int_{a}^{t}(t-\tau)^{-\alpha} f^{\prime}(\tau) d \tau, \quad 0 \leq \alpha<1
$$

Note that the kernel of (3.6) naturally appears when $\alpha$ is replaced by $1-\beta$ and it regulates the effect of the past in a $\beta$-proportional rate. The derivative value is the sum of the effect of the initial condition and the value of the integer derivative, both regulated by the kernel $h_{\infty}$. The kernel behavior is similar to the Cantor set [33], that is, an iterative function that removes the middle section of a line (Figure 4). The fractal dimension of the Cantor set is defined by

$$
D=\frac{\ln 2}{\ln 1 / \alpha}, \quad 0<\alpha<\frac{1}{2}
$$

In this case the $D$ th dimension represents the density of the remaining bars and it is analogous to the kernel $h_{\infty}$, but in discrete time. 


\section{Engineering Applications}

Fractional order can represent systems with high-order dynamics and complex nonlinear phenomena using few coefficients $[6,34,35]$, since the arbitrary order of the derivatives gives an additional degree of freedom to fit an specific behavior. Another important characteristic is that the fractional order derivatives depend not only on local conditions of the evaluated time, but also on all the history of the function. This fact is often useful when the system has long-term "memory" and any evaluation point depends on the past values of the function. However, it is also a problem when fractional derivative functions are implemented in logical circuits, because they require a huge quantity of physical memory. The strategies to simulate fractional order systems are classified in three groups [36,37].

(i) Computational methods based on the analytic equation. These methods present multiple parameters and are complicated to analyze, as it is necessary to evaluate every single point in the function and its history; moreover, the explicit equation if often difficult to obtain.

(ii) Approximation through a rational system in discrete time. The analytical system is replaced by its discrete equivalent in frequency space. Those methods result in irrational coefficients, that are approximated again by truncating the polynomial series, which is equivalent to truncate the model in the time domain; therefore, it requires as minimum the same number of coefficients as samples, losing the characteristic of "infinite memory". On the other hand, if the series has a lot of coefficients, it limits simulation in real time, as it requires more processing cycles.

(iii) Approximation of the fractional system using rational function in continuous time. This is approximated by rational continuous approach, but the series must be truncated; therefore, it must be limited to a specific frequency range of operation.

\subsection{Electronics Applications}

Another way to obtain the response of a fractional order system is by using analogical circuits with fractional order behavior as shown in Figure 5 or systems with fractal configuration as shown in Figure 6(a). Here three methods are introduced.

(i) Component by component implementation $[29,32]$. The approximation of the transfer function is done by the recursive circuit shown in Figure 5. The gain between $V_{o}$ and $V_{i}$ in Laplace transform is the continuous fraction approximation to the original system [38], that is

$$
\frac{V_{o}}{V_{i}}=1+\frac{w_{n}}{\mathrm{~s}+\frac{w_{n-1}}{1+\frac{w_{n-2}}{\mathrm{~s}+\frac{w_{n-3}}{\vdots}}}},
$$

where $w_{n-2 j}=1 / R_{j} C_{j}$ and $w_{n-2 j+1}=1 /\left(R_{j+1}+C_{j}\right)$.

This circuit has two principal disadvantages: (1) it has a limited frequency band of work, and (2) this is an approximation, therefore it requires a lot of low tolerance components, depending on the accuracy required by the designer. 


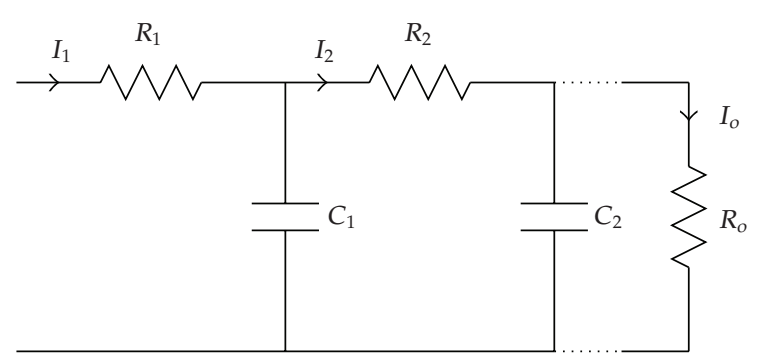

Figure 5: Recursive low pass RC filter.

(ii) Field Programmable Analog Array (FPAA) [39]. The designer implements the circuit component by component into a FPAA. It allows changing of the dynamical behavior of the fractional order system with a few simple modifications and each element has custom tolerance.

(iii) Fractional order impedance component. It is a capacitor with fractional order behavior introduced in [40]. In general it consists in a capacitor of parallel plates, where one of them presents fractal dimension (Figure 6(a)). Each branch could be modeled as a low pass resistor/capacitor (RC) circuit filter and it is linked to the principal branch, as shown in Figures 6(b) and 6(c).

Anyone of these approaches could be used in engineering applications, in this paper we introduce its use in systems' identification, control theory and robotics.

\subsection{Fractional Order Identification of Dynamical Systems}

Fractional order dynamical systems can be modeled using the Laplace transform-like transfer functions [41] as

$$
G(S)=\frac{b_{m} S^{\beta_{m}}+b_{m-1} s^{\beta_{[m-1}}+\cdots+b_{0} S^{\beta_{0}}}{a_{n} S^{\alpha_{n}}+a_{n-1} S^{\alpha_{n-1}}+\cdots+a_{0} S^{\alpha_{0}}}
$$

with $\alpha, \beta \in \Re, \alpha_{n}>\alpha_{n-1}>\cdots>\alpha_{0}$, and $\beta_{m}>\beta_{m-1}>\cdots>\beta_{0}$.

Some high-order systems would be approximated with a compact fractional order expression, it is useful in cases where an approach between holistic and detailed description of the process is required. As an instance the model of the 5th order [7]

$$
G(s)=\frac{s^{4}+36 s^{3}+126 s^{2}+84 s+9}{9 s^{4}+84 s^{3}+126 s^{2}+36 s+1} .
$$

This 8-parameter system would be well approximated by $G(s) \approx 1 / s^{0.5}$, a compact fractional order system with just a parameter, valid in the frequency range from 100 to $10000 \mathrm{~Hz}$, as shown in Figure 7.

Many real systems are better identified as fractional order equations [16, 42] than integer ones. In fact, some responses cannot be approximated just as a linear combination of exponential functions [43], and the arbitrary order is an additional degree of freedom that 


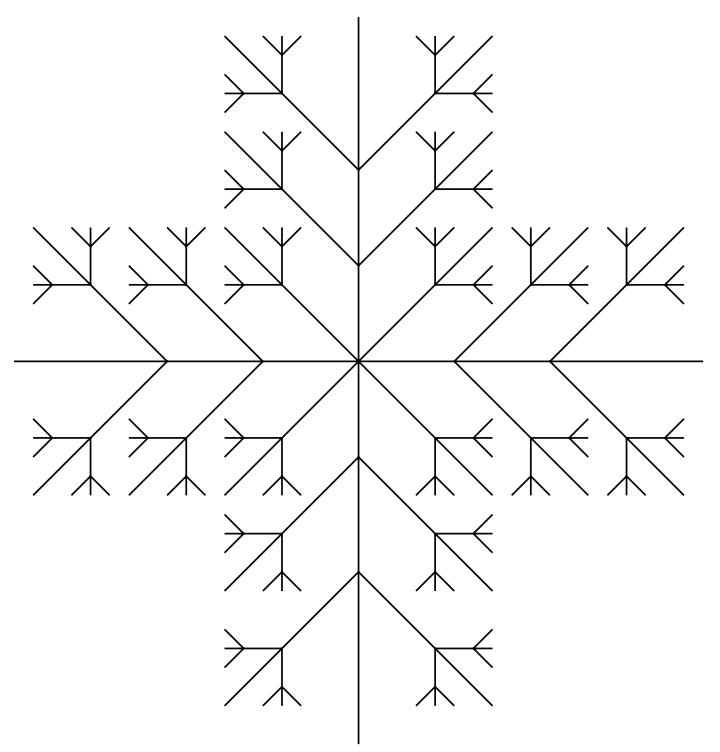

(a) Fractal tree

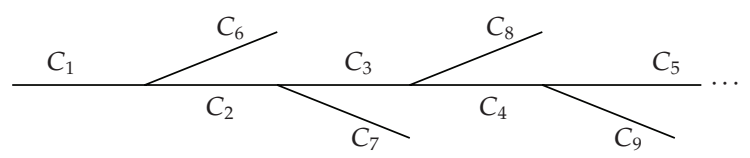

(b) Fractal branch

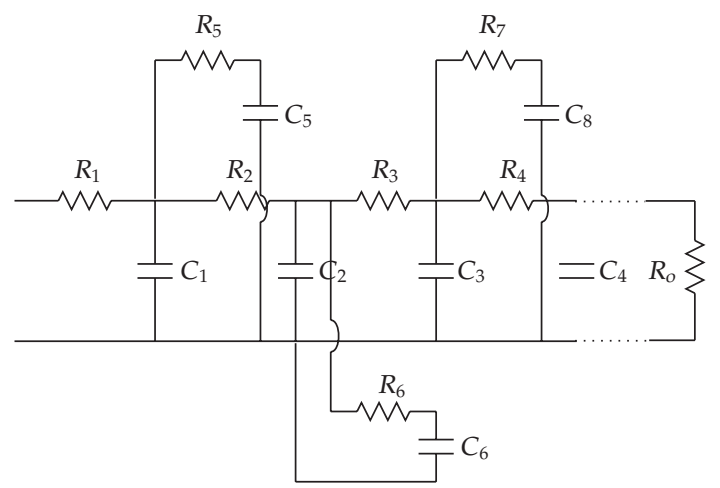

(c) Equivalent circuit of a fractal branch

Figure 6: Fractor is a parallel capacitance with fractional order behavior. It uses fractal geometry when fabricated. (a) Introducing a type of fractal tree. (b) Presenting the link diagram, and (c) The circuit equivalence.

yields a better approximation to the real system while describing it in a compact way [44]. In [45] it was used this fact to identify a fractal system, typically modeled in frequency as:

$$
F(S)=\frac{K}{s^{m}+a}, \quad m \in \mathfrak{R}, K, a \in \mathbb{Z}^{+}, s=j w .
$$




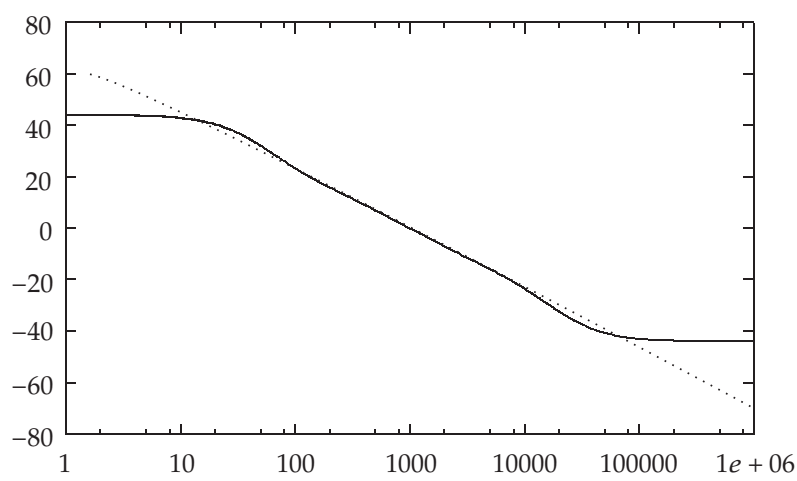

Fractional order approximation

(a) Magnitude in $\mathrm{dB}$

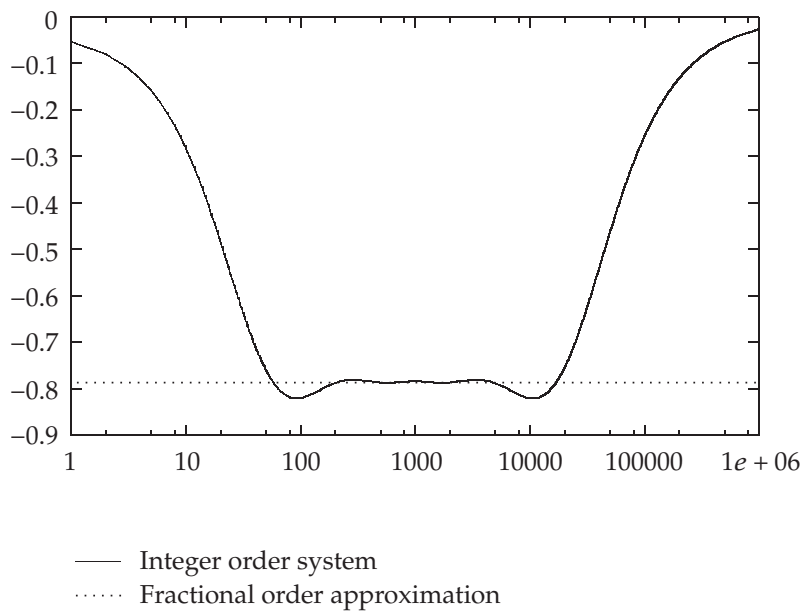

(b) Phase in $\mathrm{dB}$

Figure 7: Comparison between a high-order integer system and its approximation by a fractional one.

Adjusting the model is accomplished by finding the parameters $\{K, a, \alpha\}$ that minimize the mean error with the real data.

Another instance of the fractional order formulation is presented in [46], the authors approximated a complex system, a flexible structure with five vibration modes, modeling it with few parameters, being still valid for a wide range of frequencies. They propose the transfer function:

$$
G(S)=\frac{\sum_{i=0}^{m} a_{i}\left(S^{\alpha}\right)^{i}}{\left(S^{\alpha}\right)^{n}+\sum_{j=0}^{n-1} b_{j}\left(S^{\alpha}\right)^{j}}
$$

with $\alpha=1, \alpha=2$, and $\alpha=0.5$. A real value of $\alpha$ models the damper behavior without increasing the order of the system, and maintaining a compact expression too, valid for the frequency range $[0.1 \mathrm{~Hz}-200 \mathrm{~Hz}]$. 


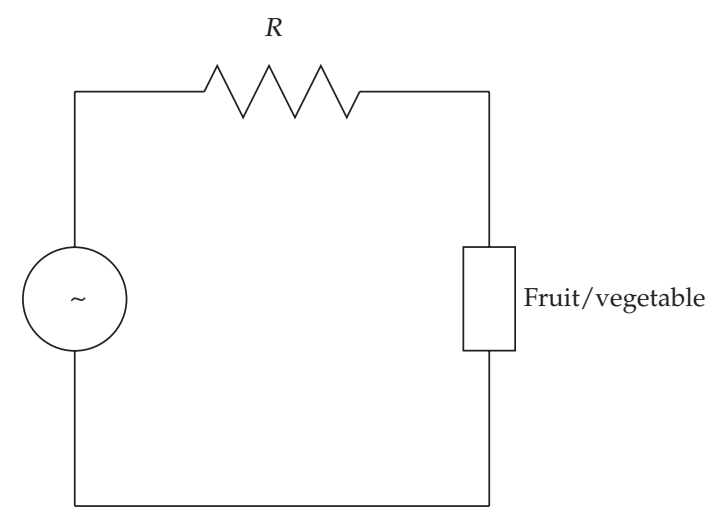

Figure 8: Circuit used for identification of fractance in fruits and vegetables.

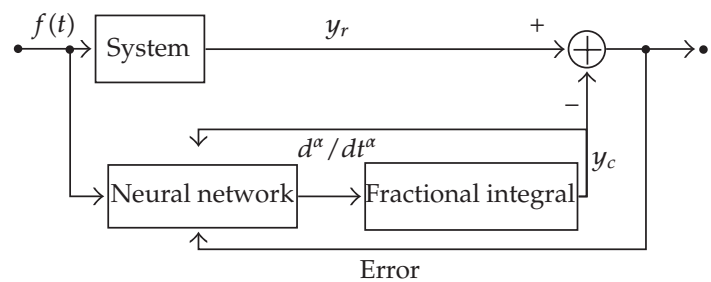

Figure 9: Block diagram of the identification of a system by CNN. Two sorts of systems would be identified, the neural network and the continuous fractional system.

Another example of identification of a biological system was presented in [20], the authors note that their frequency response does not decay/increase in multiples of $20 \mathrm{~dB} / \mathrm{dec}$ in the Bode's plot. It may occurs because fruit and vegetable's electrical properties depends on several parameters as type of fruit/vegetable, size, temperature, and pressure between others. As the author demonstrate by the experiment shown in Figure 8, by applying a sine voltage and analyzing the current over the object. They found that the response in frequency has a fractional order behavior with a constant slope, depending on the fruit/vegetable.

A nonparametric method introduced in [47] uses a continuous neural network (CNN) in order to identify nonlinear systems. This type of networks uses integral blocks instead of time delays. This fact makes the model continuous and its behavior is not a "black box" anymore. From this kind of network is possible to separate the static nonlinear system (neural network) from the dynamical one (integral blocks). If the integral blocks are fractional order blocks, then the CNN captures the fractional behavior too. In order to train the network, the authors used the square mean error between the system output $y_{r}$ and the neural network output $y_{c}$ (Figure 9).

Just as an example, we propose an experiment with synthetic data, simulating the vibration present in a gearbox. These kinds of systems are highly complex as several frequencies and their harmonics are exited by the rotation of the axes, unbalanced pieces, meshing between gears, bearing balls interaction, backslash between pieces among others. When the system has a failure, harmonics and side-bands are added to the frequency 


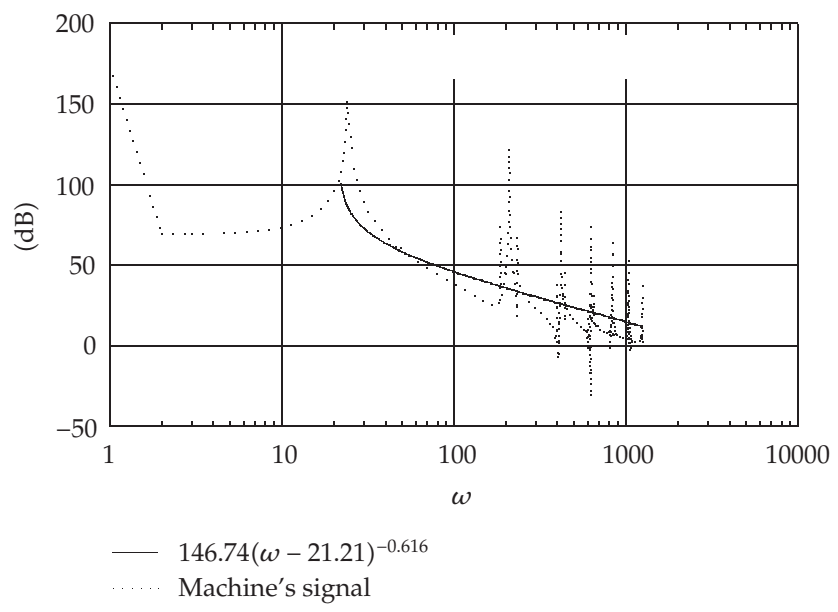

(a) System without failure

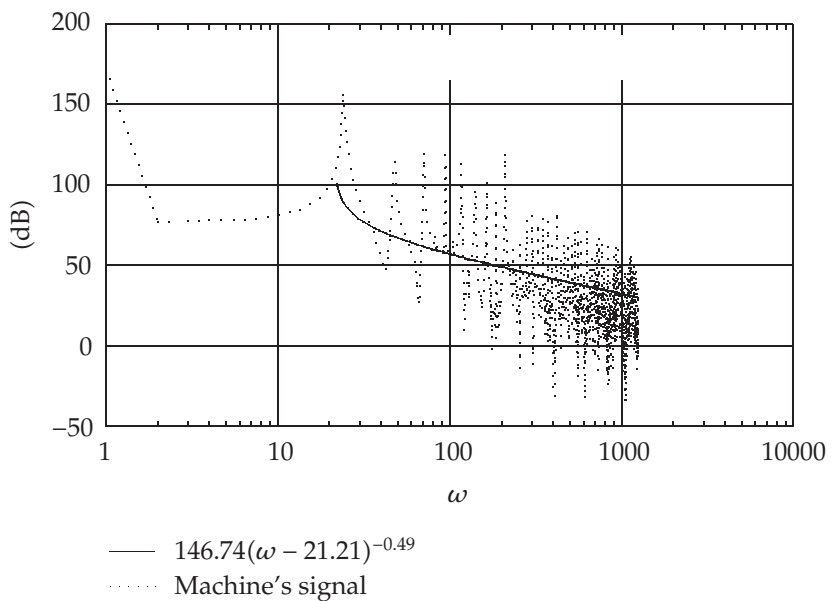

(b) System with failure

Figure 10: Magnitude of the Bode's plot of two complex systems, one represents the vibration signal of a rotational system without a failure (a) and the other is (b) a system with a teeth broken on the transmission box. Note that when approximating by a fractional order equations the order changes from a system to another.

spectrum and the dynamical model of the system may change. If these models were known a predictive maintenance strategy would be proposed based on comparison between them.

Unfortunately as there are many components interacting and some have nonlinear behavior, a dynamical model of integer order is frequently difficult to obtain and involve several parameters that are hardly comparable. Notwithstanding, as shown in Figure 10 the signal on the Bode's plot does not decay by $20 \mathrm{~dB} / \mathrm{dec}$, hence the systems would be approximate by a fractional order equations. When a failure is introduced, the model of the system change. In this case the failure was identified with just one parameter, the order of the equation. 
Table 1: Classification of dynamical system grouping by order of the plant and the controller.

\begin{tabular}{lc}
\hline Order of system & Order of controller \\
\hline Integer & Integer \\
Integer & Fractional \\
Fractional & Integer \\
Fractional & Fractional \\
\hline
\end{tabular}

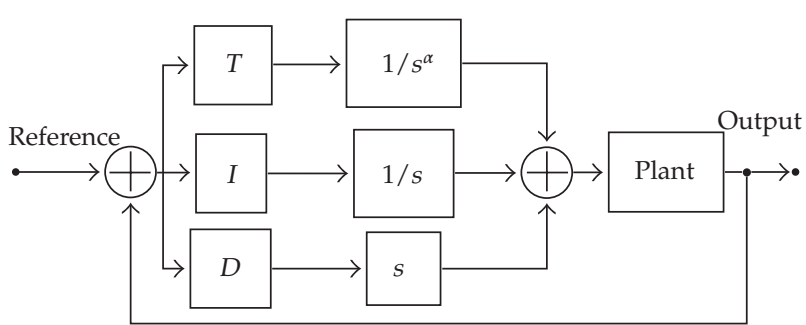

Figure 11: Block diagram of TID controller, where $0 \leq \alpha \leq 1$.

\subsection{Fractional Order Control}

Dynamic systems are typically fractional order, but often just the controller is designed as that, as the plant is modeled with integer order differintegral operators. A robust fractional order controller requires less coefficients than the integer one [48]. Grouping by type of plant and controller, the systems are classified in four sets [49], as shown in Table 1.

In [49] it is proved that fractional order controllers are more robust than integer order. The authors proposed two dynamic systems with three coefficients, (1) an integer system of second order and (2) a system of fractional order with three coefficient. They optimized those controllers and found that fractional algorithms were more stable taking into account stationary error and the overshoot percentage.

The typical fractional controller in literature are [27] as follows.

(i) Tilted Proportional and Integral (TID). It is a controller similar to the PID of integer order in its architecture, but replacing the proportional component by a function like $s^{-\alpha}$, with $\alpha \in \Re$. It gives an additional degree of freedom to the system and allows a better behavior than that of the integer order controller. A block diagram of TID controllers is shown in Figure 11.

(ii) Acronym in French of Crontrôle Robuste d'Ordre Non Entier (CRONE). These type of controllers are based on "fractal robustness" (a damping behavior that is independent of the mass observed in water dykes) [50] in which the conjugated roots of the characteristic equation of the system can move over a fixed angle in the complex plane. When analyzed in feedback, the system has a constant phase (4.8). This result is identical to the phase of the proposed system in open loop for high frequencies. Therefore, it implies that the controller is robust in this characteristic, which is directly related with the overshoot and the damper factor.

The function approximation to the dyke behavior was

$$
G(s)=\frac{1}{(\tau a)^{\alpha}+1}
$$




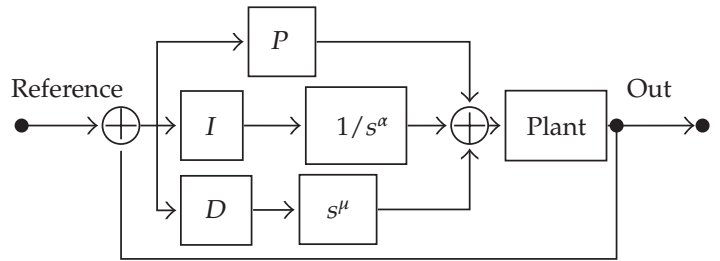

Figure 12: Block diagram of the $P I^{\alpha} D^{\mu}$ controller, where $0 \leq \alpha \leq 1$ and $0 \leq \mu \leq 1$.

Therefore, in feedback with a negative gain

$$
\begin{gathered}
G(s)=\frac{1}{e^{\alpha \ln \tau \omega}}|G(j \omega)|=e^{-\alpha \ln \tau \omega}, \\
\langle G(j \omega)\rangle=-\alpha \frac{\pi}{2} .
\end{gathered}
$$

(iii) Algorithm $P I^{\alpha} D^{\mu}$. This is the generalization of the integer PID. The general structure of this kind of controllers is

$$
\frac{O(S)}{I(S)}=P+I S^{-\alpha}+D S^{\mu}
$$

There is not a rigorous formula to design this type of controller, some techniques to adjust it are artificial intelligence, as swarm intelligence [51], genetic algorithms [52] or other where the parameter space has five variables $\left[K_{p}, K_{i}, K_{d}, \alpha, \mu\right]$. A block diagram of $P I^{\alpha} D^{\mu}$ is shown in Figure 12.

(iv) Fractional lead-lag controller. It is the generalization of the lead-lag controller of integer order. It can be written as

$$
C_{r}(S)=C_{0}\left(\frac{1+s / \omega_{b}}{1+s / \omega_{h}}\right)^{r}
$$

where $0<\omega_{b}<\omega_{h}, C_{0}>0$ and $r \in(0,1)$

In [53] the author proposes a general optimization architecture, based on Caputo formula, where the system equation is optimized in Lagrange terms as follow:

$$
\begin{gathered}
D^{\alpha} x=G(x, u, t), \\
D^{\alpha}=\frac{\delta F}{\delta x}+\lambda \frac{\delta G}{\delta x}=0
\end{gathered}
$$

where $\lambda$ is the Lagrange multiplier and the initial conditions are known.

\subsection{Applications in Robotics}

In industrial environments the robots have to execute their task quickly and precisely, minimizing production time. It requires flexible robots working in large workspaces; 


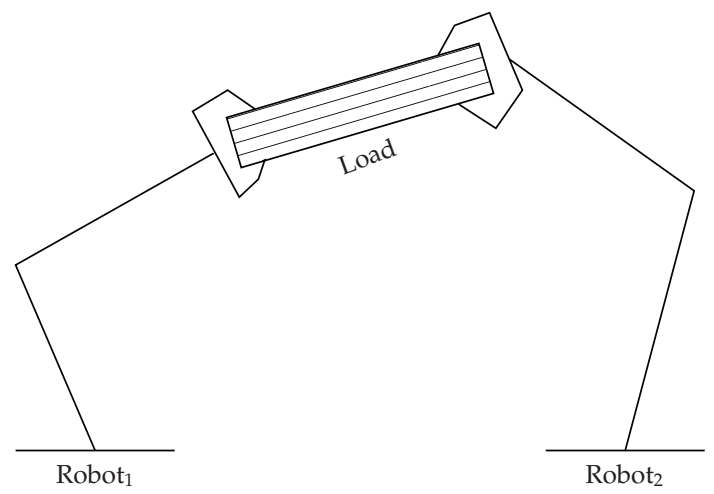

Figure 13: A cooperative cell of robots achieving a desired task.

therefore, they are influenced by nonlinear and fractional order dynamic effects [10]. For instance in $[54,55]$ the authors analyze the behavior of two links in a redundant robot (a robot that has more degree of freedom than required to carry out its task) following a circular trajectory in the Cartesian space. By calculating the inverse kinematics, the pseudoinverse matrix does not converge into an optimal solution either for repeatability or manipulability. In fact, the configuration of those links has a chaotic behavior that can be approximated by fractional order equations, since it is a phenomena that depends on the long-term history, as introduced in [56]. Another case-fractional order behavior in robotics was presented in [10], where a robot of three degrees of freedom was analyzed by following a circular trajectory, controlled with a predictive control algorithm on each joint. Despite it has an integer order model, the current of all motors at the joints presents clearly a fractional order behavior.

In [57], the authors analyze the effect of a hybrid force and position fractional controller applied to two robotic arms holding the same object, as shown in Figure 13. The load of the object varied and some disturbances are applied as reference of force and position. A $P I^{\alpha} D^{\mu}$ controller was tuned by trial and error. The resulting controller was demonstrated to be robust to variable loads and small disturbances at the reference.

Another interesting problem in robotics which can be treated, with FOC is the control of flexible robots, as this kind of light robots use low power actuators, without self-destruction effects when high impacts occurs. Nevertheless significant vibrations over flexible links make a position control difficult to design, because it reveals a complex behavior difficult to approximate by linear differential equations [58]. However in [59], the authors propose a $P D^{\alpha}$ for a flexible robot of one degree of freedom with variable load, resulting in a system with static phase and constant overshoot, independent of the applied load.

Another case was analyzed in [60], simulating a robot with two degrees of freedom, and some different physical characteristics, as an ideal robot, a robot with backslash and a robot with flexible joints. In each one of these configurations they applied PID and $P I^{\alpha} D^{\mu}$ controllers and their behavior was compared. These controllers were tuned by trial and error in order to achieve a behavior close to the ideal and tested 10000 trajectories with different type of accelerations [61]. Over the ideal robot, the PID controller had a smaller response time and smaller overshoot peak than the fractional order PID. When any kind of nonlinearity is added to the model, the fractional controller has a smaller overshoot and a smaller stationary error, demonstrating that these type of controllers are more robust than classical PID to nonlinear effects. 
A small overshoot in fractional order controllers is an important characteristic when accuracy and speed are desired in small spaces. In [62] the authors used CRONE controllers in order to reduce the overshoot on small displacement over a $X Y$ robot. The workspace is of $1 \mathrm{~mm}^{2}$ and the overshoot obtained was lower than $1 \%$.

An application in a robot with legs was presented in $[63,64]$, designing a set of $P D^{\alpha}$ algorithms in order to control position and force, applied to an hexapod robot with 12 degrees of freedom. The authors defined two performance metrics, one for quantity of energy and the other for position error. The controllers with $\alpha=0.5$ had the best performance in this robot.

\section{Conclusions}

In this paper some basic concepts of FOC and some applications in engineering were presented. However, its inherent complexity, the lack of a clear geometrical interpretation and the apparent sufficiency of the integer calculus have delayed its use outside the area of mathematics. Nowadays, some applications have begun to appear but they are still at the initial stage of development. In the near future, with a deep understanding of FOC's implications, its use in systems' identification will increase, as it captures very complex behavior neglected by IOC, and in control of systems this tool open a wide range of desired behavior, where the integer one is just a special case.

\section{Acknowledgment}

The authors acknowledge support received from the Universidade Estadual de CampinasUNICAMP (Brazil), Intituto Superior de Engenharia do Porto-I.S.E.P. (Portugal), and Coordenação de Aperfeiçoamento de Pessoal de Nível Superior-CAPES (Brazil), that made this study be possible.

\section{References}

[1] J. A. T. Machado, "A probabilistic interpretation of the fractional-order differentiation," Fractional Calculus and applied Analysis, vol. 6, no. 1, pp. 73-80, 2003.

[2] Q.-S. Zeng, G.-Y. Cao, and X.-J. Zhu, "The effect of the fractional-order controller's orders variation on the fractional-order control systems," in Proceedings of the 1st International Conference on Machine Learning and Cybernetics, vol. 1, pp. 367-372, 2002.

[3] R. L. Magin and M. Ovadia, "Modeling the cardiac tissue electrode interface using fractional calculus," Journal of Vibration and Control, vol. 14, no. 9-10, pp. 1431-1442, 2008.

[4] L. Sommacal, P. Melchior, A. Oustaloup, J.-M. Cabelguen, and A. J. Ijspeert, "Fractional multi-models of the frog gastrocnemius muscle," Journal of Vibration and Control, vol. 14, no. 9-10, pp. 1415-1430, 2008.

[5] N. Heymans, "Dynamic measurements in long-memory materials: fractional calculus evaluation of approach to steady state," Journal of Vibration and Control, vol. 14, no. 9-10, pp. 1587-1596, 2008.

[6] J. De Espíndola, C. Bavastri, and E. De Oliveira Lopes, "Design of optimum systems of viscoelastic vibration absorbers for a given material based on the fractional calculus model," Journal of Vibration and Control, vol. 14, no. 9-10, pp. 1607-1630, 2008.

[7] B. T. Krishna and K. V. V. S. Reddy, "Active and passive realization of fractance device of order $1 / 2$," Active and Passive Electronic Components, vol. 2008, Article ID 369421, 5 pages, 2008.

[8] Y. Pu, X. Yuan, K. Liao, et al., “A recursive two-circuits series analog fractance circuit for any order fractional calculus," in ICO20: Optical Information Processing, vol. 6027 of Proceedings of SPIE, pp. 509519, August 2006. 
[9] M. F. M. Lima, J. A. T. Machado, and M. Crisóstomo, “Experimental signal analysis of robot impacts in a fractional calculus perspective," Journal of Advanced Computational Intelligence and Intelligent Informatics, vol. 11, no. 9, pp. 1079-1085, 2007.

[10] J. Rosario, D. Dumur, and J. T. Machado, "Analysis of fractional-order robot axis dynamics," in Proceedings of the 2nd IFAC Workshop on Fractional Differentiation and Its Applications, vol. 2, July 2006.

[11] L. Debnath, "Recent applications of fractional calculus to science and engineering," International Journal of Mathematics and Mathematical Sciences, vol. 2003, no. 54, pp. 3413-3442, 2003.

[12] G. W. Bohannan, "Analog fractional order controller in temperature and motor control applications," Journal of Vibration and Control, vol. 14, no. 9-10, pp. 1487-1498, 2008.

[13] J. Cervera and A. Baños, "Automatic loop shaping in QFT using CRONE structures," Journal of Vibration and Control, vol. 14, no. 9-10, pp. 1513-1529, 2008.

[14] R. Panda and M. Dash, "Fractional generalized splines and signal processing," Signal Processing, vol. 86, no. 9, pp. 2340-2350, 2006.

[15] Z.-Z. Yang and J.-L. Zhou, "An improved design for the IIR-type digital fractional order differential filter," in Proceedings of the International Seminar on Future BioMedical Information Engineering (FBIE '08), pp. 473-476, December 2008.

[16] I. Petráš, "A note on the fractional-order cellular neural networks," in Proceedings of the International Joint Conference on Neural Networks, pp. 1021-1024, July 2006.

[17] L. Dorcak, I. Petras, I. Kostial, and J. Terpak, "Fractional-order state space models," in Proceedings of the International Carpathian Control Conference, pp. 193-198, 2002.

[18] D. Cafagna, "Past and present-fractional calculus: a mathematical tool from the past for present engineers," IEEE Industrial Electronics Magazine, vol. 1, no. 2, pp. 35-40, 2007.

[19] A. Benchellal, T. Poinot, and J.-C. Trigeassou, "Fractional modelling and identification of a thermal process," in Proceedings of the 2nd IFAC Workshop on Fractional Differentiation and Its Applications, vol. 2, July 2006.

[20] I. S. Jesus, J. A. T. Machado, and J. B. Cunha, "Fractional electrical dynamics in fruits and vegetables," in Proceedings of the 2nd IFAC Workshop on Fractional Differentiation and Its Applications, vol. 2, July 2006.

[21] W. M. Ahmad and R. El-Khazali, "Fractional-order dynamical models of love," Chaos, Solitons and Fractals, vol. 33, no. 4, pp. 1367-1375, 2007.

[22] A. Oustaloup, J. Sabatier, and X. Moreau, "From fractal robustness to the CRONE approach," in Systèmes Différentiels Fractionnaires (Paris, 1998), vol. 5, pp. 177-192, SIAM, Paris, France, 1998.

[23] I. Podlubny, "The laplace transform method for linear differential equations of the fractional order," Tech. Rep., Slovak Academy of Sciences, Institute of Experimental Physics, 1994.

[24] D. Xue, C. Zhao, and Y. Chen, "A modified approximation method of fractional order system," in Proceedings of the IEEE International Conference on Mechatronics and Automation (ICMA '06), pp. 10431048, 2006.

[25] J. L. Adams, T. T. Hartley, and C. F. Lorenzo, "Fractional-order system identification using complex order-distributions," in Proceedings of the 2nd IFAC Workshop on Fractional Differentiation and Its Applications, vol. 2, July 2006.

[26] M. D. Ortigueira, J. A. T. Machado, and J. S. Da Costa, "Which differintegration?" IEE Proceedings: Vision, Image and Signal Processing, vol. 152, no. 6, pp. 846-850, 2005.

[27] D. Xue and Y. Q. Chen, "A comparative introduction of four fractional order controllers," in Proceedings of the 4th World Congress on Intelligent Control and Automation, vol. 4, pp. 3228-3235, 2002.

[28] R. L. Magin and M. Ovadia, "Modeling the cardiac tissue electrode interface using fractional calculus," in Proceedings of the 2nd IFAC Workshop on Fractional Differentiation and Its Applications, vol. 2, July 2006.

[29] K. B. Oldham and J. Spanier, The Fractional Calculus: Theory and Applications of Differentiation and Integration to Arbitrary Order, vol. 1, Dover, New York, NY, USA, 2006.

[30] C. Ma and Y. Hori, "Fractional order control and its application of PI $\alpha$ D controller for robust twoinertia speed control," in Proceedings of the 4th International Power Electronics and Motion Control Conference (IPEMC'04), vol. 3, pp. 1477-1482, August 2004.

[31] I. Podlubny, "Geometric and physical interpretation of fractional integration and fractional differentiation," Fractional Calculus E Applied Analysis, vol. 5, no. 4, pp. 367-386, 2002.

[32] M. Moshrefi-Torbati and J. K. Hammond, "Physical and geometrical interpretation of fractional operators," Journal of the Franklin Institute, vol. 335, no. 6, pp. 1077-1086, 1998.

[33] S. Miyazima, Y. Oota, and Y. Hasegawa, "Fractality of a modified Cantor set and modified Koch curve," Physica A, vol. 233, no. 3-4, pp. 879-883, 1996. 
[34] F. B. M. Duarte and J. A. T. Machado, "Fractional dynamics in the describing function analysis of nonlinear friction," in Proceedings of the 2nd IFAC Workshop on Fractional Differentiation and Its Applications, vol. 2, July 2006.

[35] P. J. Torvik and R. L. Bagley, "On the appearance of the fractional derivative in the behaviour of real materials," Journal of Applied Mechanics, vol. 51, no. 2, pp. 294-298, 1984.

[36] D. Baleanu and S. I. Muslih, "Nonconservative systems within fractional generalized derivatives," Journal of Vibration and Control, vol. 14, no. 9-10, pp. 1301-1311, 2008.

[37] T. Poinot and J.-C. Trigeassou, "A method for modelling and simulation of fractional systems," Signal Processing, vol. 83, no. 11, pp. 2319-2333, 2003.

[38] S. Oh and Y. Hori, "Realization of fractional order impedance by feedback control," in Proceedings of the 33rd Annual Conference of the IEEE Industrial Electronics Society (IECON '07), pp. 299-304, 2007.

[39] R. Caponetto and D. Porto, "Analog implementation of non integer order integrator via field programmable analog array," in Proceedings of the 2nd IFAC Workshop on Fractional Differentiation and Its Applications, vol. 2, July 2006.

[40] T. C. Haba, G. Ablart, T. Camps, and F. Olivie, "Influence of the electrical parameters on the input impedance of a fractal structure realised on silicon," Chaos, Solitons and Fractals, vol. 24, no. 2, pp. 479-490, 2005.

[41] I. Podlubny, I. Petráš, B. M. Vinagre, P. O’Leary, and L. Dorčák, “Analogue realizations of fractionalorder controllers," Nonlinear Dynamics, vol. 29, no. 1-4, pp. 281-296, 2002.

[42] J. J. De Espiíndola, J. M. Da Silva Neto, and E. M. O. Lopes, "A generalised fractional derivative approach to viscoelastic material properties measurement," Applied Mathematics and Computation, vol. 164, no. 2, pp. 493-506, 2005.

[43] B. M. Vinagre and V. Feliu, "Optimal fractional controllers for rational order systems: a special case of the Wiener-Hopf spectral factorization method," IEEE Transactions on Automatic Control, vol. 52, no. 12, pp. 2385-2389, 2007.

[44] T. T. Hartley and C. F. Lorenzo, "Fractional-order system identification based on continuous orderdistributions," Signal Processing, vol. 83, no. 11, pp. 2287-2300, 2003.

[45] A. Djouambi, A. Charef, and A. V. Besan, "Approximation and synthesis of non integer order systems," in Proceedings of the 2nd IFAC Workshop on Fractional Differentiation and Its Applications, vol. 2, July 2006.

[46] B. M. Vinagre, V. Feliu, and J. J. Feliu, "Frequency domain identification of a flexible structure with piezoelectric actuators using irrational transfer function models," in Proceedings of the 37th IEEE Conference on Decision and Control, vol. 17, pp. 1278-1280, December 1998.

[47] F. Benoit-Marand, L. Signac, T. Poinot, and J.-C. Trigeassou, "Identification of non linear fractional systems using continuous time neural networks," in Proceedings of the 2nd IFAC Workshop on Fractional Differentiation and Its Applications, vol. 2, July 2006.

[48] D. Xue, C. Zhao, and Y. Chen, "Fractional order PID control of A DC-motor with elastic shaft: a case study," in Proceedings of the American Control Conference, pp. 3182-3187, 2006.

[49] Y. Chen, "Ubiquitous fractional order controls?" in Proceedings of the 2nd IFAC Workshop on Fractional Differentiation and Its Applications, vol. 2, July 2006.

[50] A. Oustaloup, J. Sabatier, and X. Moreau, "From fractal robustness to the CRONE approach," in Fractional Differential Systemas: Models, Methods and Applications, vol. 5, pp. 177-192, SIAM, Paris, France, 1998.

[51] N. Sadati, M. Zamani, and P. Mohajerin, "Optimum design of fractional order PID for MIMO and SISO systems using particle swarm optimization techniques," in Proceedings of the 4th IEEE International Conference on Mechatronics (ICM '07), pp. 1-5, 2007.

[52] J. Y. Cao, J. Liang, and B.-G. Cao, "Optimization of fractional order PID controllers based on genetic algorithms," in Proceedings of the 4th International Conference on Machine Learning and Cybernetics, pp. 5686-5689, 2005.

[53] O. P. Agrawal, "A formulation and a numerical scheme for fractional optimal control problems," in Proceedings of the 2nd IFAC Workshop on Fractional Differentiation and Its Applications, vol. 2, July 2006.

[54] F. B. M. Duarte, M. da Graça Marcos, and J. A. T. Machado, "Fractional-order harmonics in the trajectory control of redundant manipulators," in Proceedings of the 2nd IFAC Workshop on Fractional Differentiation and Its Applications, vol. 2, July 2006.

[55] F. B. M. Duarte and J. A. Machado, "Pseudoinverse trajectory control of redundant manipulators: a fractional calculus perspective," in Proceedings of the IEEE International Conference on Robotics and Automation, vol. 3, pp. 2406-2411, 2002. 
[56] F. B. M. Duarte and J. A. T. Machado, "Chaotic phenomena and fractional-order dynamics in the trajectory control of redundant manipulators," Nonlinear Dynamics, vol. 29, no. 1-4, pp. 315-342, 2002.

[57] N. M. F. Ferreira and J. A. T. Machado, "Fractional-order position/force control of two cooperating manipulators," in Proceedings of the IEEE International Conference on Computational Cybertnetics, August 2003.

[58] M. F. M. Lima, J. T. Machado, and M. Cris, "Fractional order fourier spectra in robotic manipulators with vibrations," in Proceedings of the 2 nd IFAC Workshop on Fractional Differentiation and Its Applications, vol. 2, July 2006.

[59] C. A. Monje, F. Ramos, V. Feliu, and B. M. Vinagre, “Tip position control of a lightweight flexible manipulator using a fractional order controller," IET Control Theory and Applications, vol. 1, no. 5, pp. 1451-1460, 2007.

[60] N. M. F. Ferreira and J. A. T. Machado, "Fractional-order hybrid control of robotic manipulators," in Proceedings of the 11th International Conference on Advanced Robotics, pp. 393-398, 2003.

[61] N. M. F. Ferreira, J. A. T. Machado, A. M. S. F. Galhano, and J. B. Cunha, "Fractional control of two arms working in cooperation," in Proceedings of the 2nd IFAC Workshop on Fractional Differentiation and Its Applications, vol. 2, July 2006.

[62] B. Orsoni, P. Melchior, A. Oustaloup, Th. Badie, and G. Robin, "Fractional motion control: application to an XY cutting table," Nonlinear Dynamics, vol. 29, no. 1-4, pp. 297-314, 2002.

[63] M. F. Silva and J. A. T. MacHado, "Fractional order PD ${ }^{\alpha}$ joint control of legged robots," Journal of Vibration and Control, vol. 12, no. 12, pp. 1483-1501, 2006.

[64] M. F. Silva, J. A. T. Machado, and R. S. Barbosa, "Comparison of different orders pad fractional order pd0.5 control algorithm implementations," in Proceedings of the 2nd IFAC Workshop on Fractional Differentiation and Its Applications, vol. 2, July 2006. 


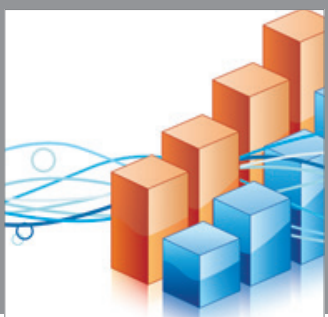

Advances in

Operations Research

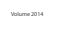

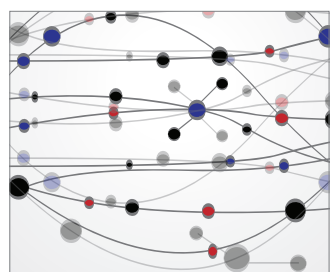

\section{The Scientific} World Journal
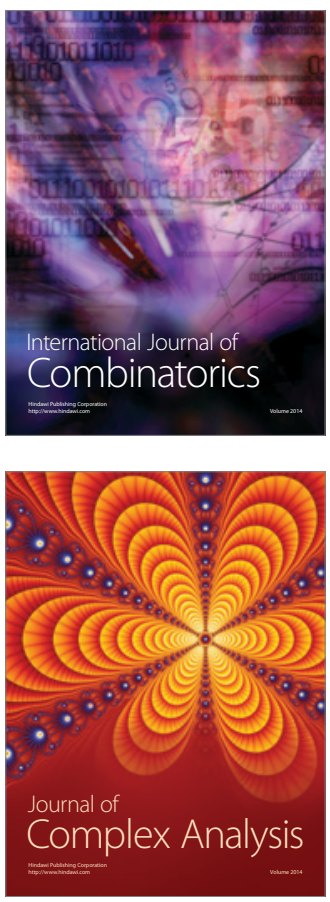

International Journal of

Mathematics and

Mathematical

Sciences
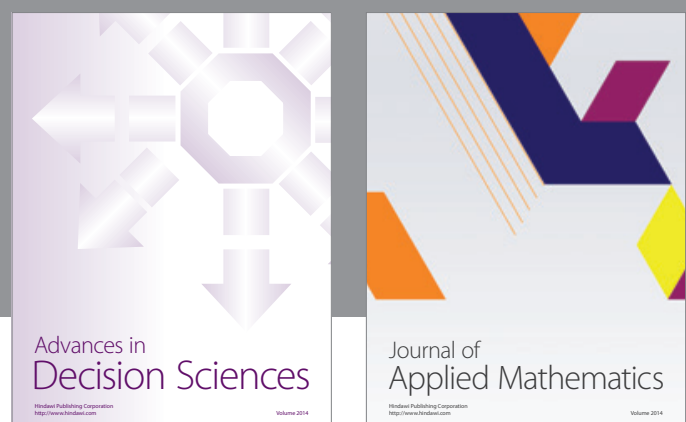

Journal of

Applied Mathematics
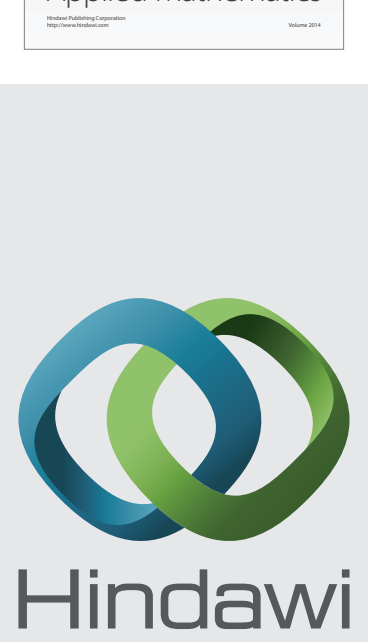

Submit your manuscripts at http://www.hindawi.com
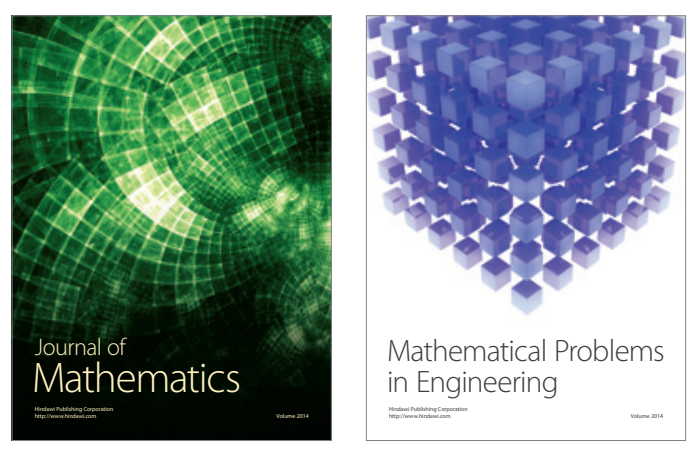

Mathematical Problems in Engineering
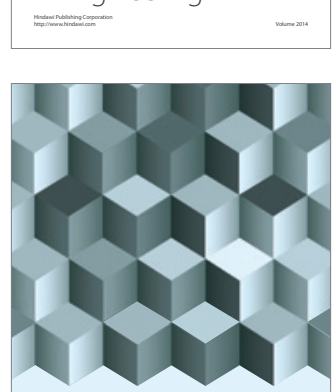

Journal of

Function Spaces
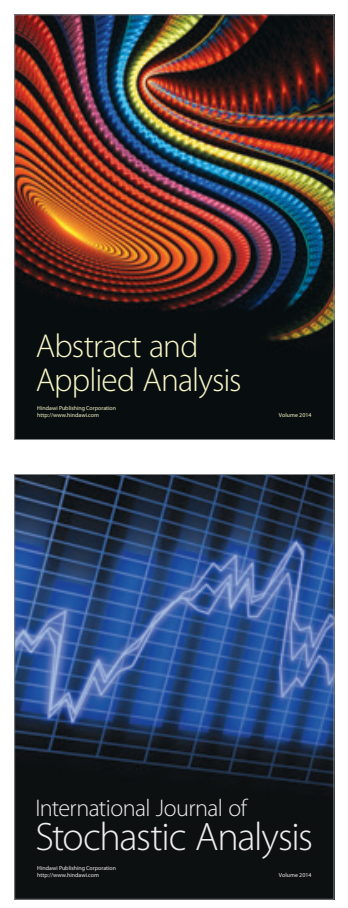

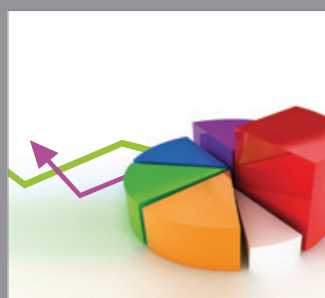

ournal of

Probability and Statistics

Promensencen
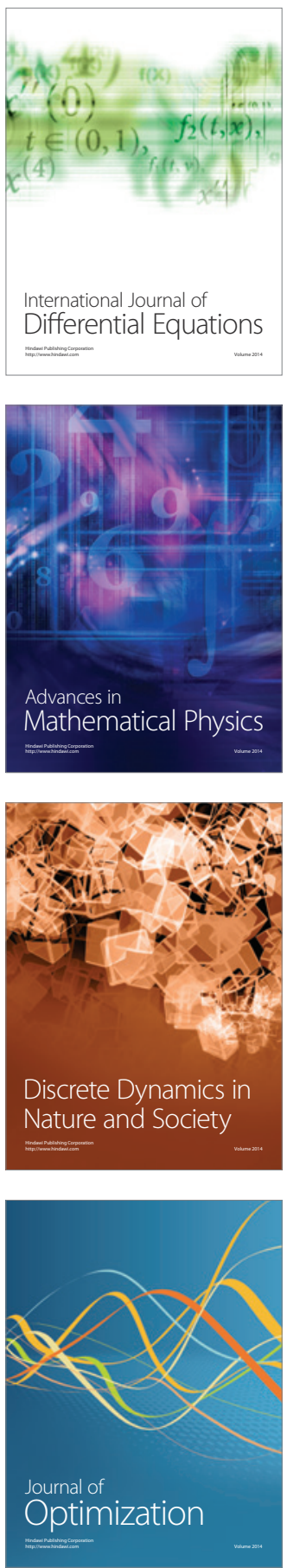